\title{
Ampliando fronteras: el derecho y la ley como ejercicios discursivos e ideológicos en la cotidianidad
}

\author{
Gonzalo Albornoz Barra \\ Universidad de la Frontera de Temuco, Chile \\ gonzaloalbornozbarra@gmail.com
}

\begin{abstract}
RESUMEN
Considerando que el derecho y la norma son producciones culturales, la visión crítica y multidisciplinaria en el estudio del derecho no solo significa un aporte vinculado al mundo de las Ciencias Sociales y Humanidades, muy por el contrario, los enfoques multidisciplinarios en el estudio de lo jurídico pueden tener importantes efectos sociales, ayudando a comprender y explicar de forma más exacta las implicancias de la ley en la vida cotidiana de todos los sujetos. Las siguientes páginas intentarán defender la idea de que el derecho y la ley son a la vez, producciones culturales y dispositivos de control que operan con una libertad peligrosa ante la ausencia de una mirada crítica sobre el carácter y los efectos del derecho en la vida cotidiana y en las representaciones que se construyen sobre el mundo.
\end{abstract}

Palabras clave: Multidisciplinariedad, derecho, representaciones sociales, producción cultural.

\section{Extending borders: law and the law as discursive and ideological exercises in the everydayness}

\begin{abstract}
Considering that the right and the norm are cultural productions, the critical and multidisciplinary vision in the study of law not only means a contribution linked to the world of Social Sciences and Humanities, on the contrary, multidisciplinary approaches in the study of the legal can have important social effects, helping to understand and explain more accurately the implications of the law in the daily lives of all subjects. The following pages will try to defend the idea that the right and the law are at the same time, cultural productions and control devices that operate with a dangerous freedom in the absence of a critical look at the character and effects of law in everyday life and in the representations that are built in the world.
\end{abstract}

KEYwORDs: Multidisciplinarity, right, social representations, cultural production. 


\section{Más allá de lo jurídico: la importancia de la multidisciplinariedad en el estudio del derecho y la ley}

Mientras que el derecho es una disciplina jurídico-social, la ley es - entre otras cosas un instrumento ideológico; siendo ambos a la vez ejercicios culturales y discursivos que operan en la práctica cotidiana de los actores. Estas nociones sobre ley y derecho, intentan poner de relieve la cada vez más patente necesidad de ampliar las fronteras críticas de la reflexión jurídica y sus alcances sociales.

El Derecho es una disciplina jurídico-social porque para operar, necesita referirse a lo humano, y en tanto, a lo social como instinto gregario del individuo. Esto queda referido en variadas nociones teóricas sobre el derecho y la ley de clásicos de la literatura jurídica como Hans Kelsen, que en General Theory of Law and State menciona que:

El derecho es un orden de la conducta humana. Un 'orden' es un conjunto de normas. El derecho no es, como a veces se dice, una norma. Es un conjunto de normas que tienen el tipo de unidad a que nos referimos cuando hablamos de un sistema» (Kelsen, 1945, p. 3).

También, desde un enfoque opuesto a la idea Kelseniana de Derecho, la noción Tomista $^{1}$ hace una referencia similar cuando menciona que: [...] «el fundamento del derecho es la naturaleza humana» (Riofrío, 2013, p. 67). Se podrían seguir citando autores bajo esta noción de Derecho, sin embargo, dos cosas parecen claras cuando se revisan diversos tratados de la Teoría General del Derecho: a) que el intento por dar con una definición del mismo es algo a lo menos ambicioso; y b) que el Derecho es un fenómeno humano y, en tanto, social.

Por otro lado, la Ley es un instrumento ideológico porque su construcción como norma regulatoria y coercitiva, se da como una consecuencia de contextos culturales, políticos y sociales determinados, principalmente, por un sistema de ideas en constante conflicto según intereses colectivos y particulares en espacios de ejercicio del poder. Así, las Leyes modernas no son otra cosa que la expresión y el resultado de discusiones políticas al interior de un parlamento en el cual, por cierto, no necesariamente se verán representados los intereses y necesidades de la sociedad.

Teniendo en cuenta que la política es, aún de forma ambigua: «el arte de gobernar con el consentimiento de los hombres» (Platón en Silva Bascuñan, 1997, p. 34), y considerando la idea weberiana de que la política es en principio una lucha (Nosetto, 2015); se debe tener en cuenta que el ejercicio político no responde a la representativi-

1 Referencias a la noción de Derecho y Ley sostenida por Santo Tomás de Aquino en Juan Carlos Riofrío, (2013). «Lecturas jurídicas de la obra de Santo Tomás de Aquino». Revista Dikaion no 1 Vol. 22. Universidad de la Sabana. Quito, Ecuador. 
dad de ideas, sino más bien a la capacidad de ciertos grupos en condición de poder para representar con un mayor alcance las ideologías que dan coherencia a sus intereses. Así, algunos aspectos que determinan la discusión legislativa responden a: la contingencia, los intereses particulares, partidistas, los intereses corporativos y de grupos, la captación de votantes, la presión internacional, etc.

Con todo, si la Ley es un instrumento determinado por el ejercicio legislativo en dicho contexto pugna política, es en también una expresión del poder oculta bajo una institucionalidad que la reviste como herramienta regulatoria y social aparentemente desprovista de subjetividad. Al respecto Foucault señala:

El derecho es la máscara del poder, así se definen los mecanismos por los cuales el poder se manifiesta, ya que es el mismo Derecho el cual delimita y enmarca de una manera formal al poder. Esta relación donde el poder se manifiesta produce consecuentemente efectos de verdad. Toda práctica de poder produce verdad, y toda verdad produce poder (Foucault en Aguilera y González, 2011 P. 06).

En el campo de reflexión sobre las implicancias del derecho y la ley la posición de los juristas es privilegiada, presentándose como aquella disciplina dotada de las capacidades necesarias para recrear los alcances más profundos y esenciales de la acción jurídica. En ella, el aporte crítico de otras ciencias o disciplinas parece algo menos relevante. Giménez expresa esta realidad cuando dice que aunque no se suele negar la pertinencia y utilidad cultural de otros enfoques respecto de la ley, los juristas, estudiosos del derecho:

[...] lo consideran como un punto de vista 'externo', que en el mejor de los casos se detiene en los suburbios del fenómeno jurídico, sin poder avanzar hasta su mismo centro... En otras palabras, el Derecho sería autónomo y autosuficiente, encontraría explicación en si mismo, y sólo sería susceptible de un análisis inmanente que no tendría por qué remitirse a elementos extra jurídicos (Giménez, 1981, p. 65).

El desplazamiento de la reflexión de otras ciencias hacia un lugar menos relevante en el discurso de los juristas debe arrojar por lo pronto dos conclusiones: a) la presencia de una dicotomía que caracteriza a lo jurídico en el centro, y a los estudios culturales del derecho y la ley en la periferia; y b) el hecho de que siendo el derecho y la ley productos socio-culturales, precisan de la reflexión multidisciplinaria, la cual daría valor al ejercicio jurídico y sus alcances como expresiones del poder político.

Por ejemplo, en un conocido manual sobre Teoría general del Derecho varios alumnos de Derecho en universidades chilenas estudian la definición de las normas jurídicas bajo la idea que: 
La norma jurídica no es más que el conjunto de reglas o preceptos que se imponen a la conducta de los seres humanos que viven en sociedad y cuyo cumplimiento puede ser coactivamente (obligatoriamente) exigido en la mayor parte de las cosas (Escandón, 2008).

Este discurso es erróneo. Cuando se dice que «la norma jurídica no es más que», se deja de lado el carácter cultural, social y político que inspira la construcción del precepto legal en contextos del ejercicio del poder. Cuando definiciones así se reproducen por años en la enseñanza de distintas escuelas de Derecho, se cae - intencionalmente o no - en una práctica que sustenta y avala científica e institucionalmente la errada caracterización de la Ley como instrumento objetivo de regulación social.

Lo anterior es preocupante porque la ley efectivamente, manda su cumplimiento obligatorio, lo que hace que pueda transformarse en un instrumento avalado institucionalmente, que opere en favor de poderes e interés corporativos, o que genere representaciones falsas de verdad desplazando los intereses y la necesidad de grupos con menor poder político.

Lo dicho puede verse en distintos acontecimientos chilenos como: el cambio en la legislación bajo el gobierno del expresidente Lagos, que buscó facilitar las condiciones de operatividad de la central Española de Endesa en Ralco, región del Biobío, dañando el ecosistema y provocando el desplazamiento y cambios culturales de comunidades rurales; la implementación sostenida de la Ley 18.314, que crea condiciones de criminalización de mapuches y la Araucanía como zona terrorista; o la aprobación de una Ley de pesca y otras normas respecto a la utilización de semillas que dificultan el trabajo de pequeños pescadores y agricultores en diversas zonas de Chile.

Cuando la ley se representa como un instrumento netamente jurídico, y no se repara en sus efectos o en los intereses que la fundan como instrumento de poder, se construye un escenario propicio para la desigualdad e injusticia social que se produce a través del mismo ejercicio legal. El llamado es entonces a ampliar las fronteras críticas y metodológicas de las Ciencias Jurídicas en el conocimiento, reflexión y diseño del derecho y la ley, entendiendo que lo jurídico no se limita a aspectos técnicos sobre la norma, y más bien tiene que ver con procesos socioculturales complejos, variables y dinámicos que ameritan enfoques multidisciplinarios.

Ampliar las fronteras dando el valor adecuado a todas las ciencias en la construcción del conocimiento jurídico y en su ejercicio, no haría más que enriquecer al derecho y la ley como instrumentos de regulación, coerción, dirección moral y ética en una sociedad caracterizada por la postverdad en todos los planos, la incertidumbre y el cambio constante, o el conflicto y desigualdad a toda escala, entre muchos otros fenómenos que dibujan el escenario de los tiempos actuales. 
Dichos aportes, podrían materializarse en ejercicios legislativos más eficientes y sincrónicos con las dinámicas propias de la llamada sociedad de derecho. La mayor eficacia y eficiencia del ejercicio legislativo a través de una reflexión crítica de la ley como instrumento subjetivo y sociocultural, contribuiría a la creación y gestión de mejores políticas públicas, y a un empoderamiento ciudadano basado en la concientización de los efectos discursivos que tiene la ley en la construcción de opiniones, creencias o estereotipos, dando paso a un manejo más preciso de ésta como instrumento de coerción social.

\section{El derecho y la ley como discurso ideológico}

Las ideologías pueden entenderse como un sistema de creencias fundamental, que organiza y fundamenta las representaciones sociales compartidas por los miembros de grupos ideológicos (Van Dijk, 2005a, p. 12; 2005b, p. 18-20). Por otro lado, de Nieto en Estudios en homenaje a Marcia Muñoz de Alba Medrano rescatamos la idea que:

El Derecho no es una disciplina pura. Todo proceso que tenga que ver con la producción de normas generales, con la aplicación de estas a casos concretos, con la enseńanza y la investigación jurídica, tiene una profunda carga ideológica. Cada operador jurídico, de conformidad con sus experiencias y convicciones personales, e incluso, de acuerdo con su propia posición epistemológica, imprime a las diversas actividades del ámbito jurídico una determinada concepción del Derecho (Nieto, 2007. p. 387).

Que el Derecho sea una disciplina cargada ideológicamente no es un tema menor. El ejercicio legal se representa comúnmente como objetivo. No obstante, aquel carácter del que goza el derecho y la ley, a parte de ser errado, es también peligroso si se pasa por alto.

Teniendo en cuenta que «El poder no se aplica a los individuos, sino que transita a través de estos» (Foucault en Aguilera y González, 2011, p. 03), la ley es uno de los tantos aparatos a través del cual transita el poder bajo formas de legitimidad cubiertas de aquella falsa objetividad de la que se hace referencia. La ley como instrumento legítimo e institucionalizado, está facultada para definir los fenómenos que regula.

Se sostiene que el carácter objetivo de la ley no es tal, asumiendo que la ley es un instrumento legitimado institucional y socialmente mediante una serie de procesos en acorde con ejercicios del poder, y ajenos en la práctica de un ejercicio abierto a la construcción ciudadana y/o colectiva. La ley, aparece así como una herramienta "carente de implicaciones valorativas» (Vilajosana, 2010, p. 11), facultada por una serie de poderes políticos para su uso como instrumento útil en la definición conductas y sanciones. 
El vasto trabajo de Michel Foucault en La verdad y las formas jurídicas, entre tantos otros trabajos y clases magistrales al respecto, también entrega insumos importantes a esta noción de Ley cuando dice que las prácticas judiciales: [...] «son algunas de las formas empleadas por nuestra sociedad para definir tipos de subjetividad, formas de saber $y$, en consecuencia, relaciones entre el hombre y la verdad que merecen ser estudiadas» (Foucault, 1978, p. 5).

Las capacidades de las que goza el ejercicio jurídico, se desplazan hacia el espacio no jurídico bajo formas de uso discursivo de la ley a nivel social. Estos usos, hacen posible a los sujetos construir narrativas y delimitar las fronteras conceptuales de los fenómenos que la Ley regula en el espacio de la cotidianidad. Se pueden citar diversas formas de invocación de la ley con el objetivo de definir fenómenos; por ejemplo, esta es la respuesta espontánea de un diputado de la República de Chile ante la pregunta «¿Qué es para usted el terrorismo?»:

Bueno, yo sigo la definición legal de terrorismo, que básicamente tiene dos formas de considerar terrorismo, cuando uno ejerce violencia sobre un grupo específico de la población, por una parte, y cuando la violencia tiene como fin último arrancar una decisión de la autoridad, a mi juicio éstas dos formas se puede entender como terrorismo (Albornoz, 2018, p. 191).

$\mathrm{Al}$ respecto, hay que considerar que la Ley Antiterrorista Chilena no exibe definiciones concretas que permitan caracterizar con asertividad las conductas terroristas, por lo que «seguir la definición legal de terrorismo...» más bien responde a un uso conceptual de la ley en la construcción de ideas sobre este mismo fenómeno. En este uso, la ley parece tener un carácter incuestionable que refuerza la definición expresada por el diputado.

Con todo, hay que tener en cuenta que: «Los aparatos jurídicos son el sistema institucional especializado para la producción o aplicación del Derecho» (Giménez 1981, p. 65). Estos, a la vez que políticos — como soporte institucional— son también ideológicos — como sistema de ideas y herramientas discursivas en la formación y circulación de representaciones. El parlamento, como lugar donde se gesta la ley, es un ejemplo del espacio que comparte el carácter político/ideológico al que se hace referencia. «La hegemonía eventual de estas ideologías, dependerá de la coyuntura sociocultural, de la correlación de fuerzas que la subentiende, y del peso institucional de los aparatos que le sirven de soporte» (Giménez, 1981, p. 87).

Las ideologías que componen el ejercicio jurídico no son necesariamente sistemas de representación intencional. El conocimiento según Foucault (2014, p. 21) se construye más bien en dos planos: a) los «Saberes sometidos» como aquellos construidos y promovidos desde la institucionalidad, y b) los «Saberes de la gente» como aquella 
construcción que surge desde el devenir particular y social propio de los actores en sus diversos contextos.

Cuando se dice que la ley y el derecho son instrumentos ideológicos, no se quiere decir que las ideologías estén impresas de forma literal en la norma escrita, sino más bien que a través de sus usos para la construcción de narrativas, la ley puede transformarse en un instrumento que permita construir y legitimar ideologías, y con esto, representaciones sociales.

\section{El derecho, la ley y las representaciones sociales en la cotidianidad}

Entendiendo ideología como:

Cualquier forma socialmente procesada y socialmente eficaz de representación o esquematización de la realidad, presente por lo menos implícitamente no sólo en el discurso sino en cualquier práctica social, como una dimensión posible de análisis (...) El Derecho es también un fenómeno ideológico susceptible como tal, de un análisis específico bajo el ángulo de sus significaciones sociales (Giménez, 1981, p. 86).

La ley es un discurso, y como discurso, se transforma en un instrumento para la construcción de narrativas y producción cultural. La ley y el derecho son ejercicios que se fundan en prácticas políticas, y como expresa Van Dijk:

Las ideologías políticas no sólo están implicadas en la producción o la comprensión de los discursos políticos y otras prácticas políticas, sino que también son (re)producidas por ellos. En cierto modo, los discursos hacen «observables» las ideologías en el sentido que es sólo en el discurso que ellas pueden ser explícitamente «expresadas» y «formuladas». Otras prácticas políticas sólo muestran o experimentan implícitamente las ideologías, por ejemplo, las prácticas de discriminación sobre la base de ideologías sexistas, racistas o políticas [...] (Van Dijk, 2005b, p. 26).

Los discursos del derecho y la ley, pueden transformarse en instrumentos con la capacidad de reproducir ideologías a través de su producción y reproducción en distintas prácticas que van desde lo político y lo judicial, hasta las prácticas más triviales e informales. A su vez la ley, al estar revestida de una legitimidad conceptual casi incuestionable, sirve como instrumento para la construcción de representaciones sobre diversos fenómenos.

Las narrativas ideológicas del derecho se desplazan a la vida social como discursos mediante múltiples formas de uso y consumo determinadas, en parte, por el contexto de existencia de cada actor. Más allá, Giménez plantea que: 
En su forma codificada, el discurso de la Ley comporta obviamente una dimensión ideológica en la medida que, ya sea a nivel formal, ya sea a nivel de contenido, vehicula cierta imagen implícita o manifiesta de la realidad social. Esta aparecerá por ejemplo como un mundo poblado de sujetos jurídicos abstractos, reputados, libres o iguales, en permanente y voluntaria actividad de intercambio y comunicación (Giménez, 1981, p. 88).

Por otro lado, se puede entender a las representaciones sociales como un punto de conexión entre lo psicológico y lo social; o como menciona Jodelet en La Representación social-Fenómeno, concepto y teoría:

(...) una forma de conocimiento específico, el saber de sentido común, cuyos contenidos manifiestan la operación de procesos generativos y funcionales socialmente caracterizados. En sentido más amplio, designa una forma de pensamiento social. Las representaciones sociales constituyen modalidades de pensamiento práctico orientados hacia la comunicación, la comprensión y el dominio del entorno social, material e ideal (Jodelet, 1986, p. 474).

Teniendo en cuenta que el conocimiento no se desarrolla solo a través de la capacitación formal, sino más bien a través de un diálogo entre ésta y una educación informal mediada por las experiencias cotidianas de la vida (Foucault, 1996; 2003; 2014; De Certeau, 1986); los discursos del derecho y la ley operan en estos dos sentidos, contribuyendo a la construcción de representaciones sociales en el plano de la cotidianidad.

Las prácticas que expresan en mayor parte los usos del Derecho y la Ley como discurso ideológico en la construcción de representaciones sociales son principalmente de uso conceptual y argumentativas: a) Sentidos comunes del derecho y la ley como sinónimos de justicia o; b) Construcciones argumentativas que utilizan al derecho y la ley para definir, calificar, tipificar, clasificar, etcétera (Giménez, 1981, p. 89; Albornoz, 2018).

Complementando, de la teoría de Michel De Certeau (1986) podemos extraer que los actores producen y reproducen interpretaciones sobre el mundo a través de, básicamente, tres procesos: a) El consumo en su amplia definición; b) La producción propia de cada actor basado en lo consumido; y c) La reproducción de aquellas producciones propias, que a la vez dan paso a nuevas prácticas de consumo para otros sujetos o comunidades.

Así, de la dinámica denominada Producción de los consumidores (De Certeau, 1986) se puede construir al menos la hipótesis de que, las ideologías y representaciones que constituyen a las leyes y al derecho debiesen tener algún grado de presencia en las interpretación, definición o caracterización de fenómenos que los sujetos hagan desde el 
ámbito de la cotidianidad. Esta presencia en la construcción de representaciones estará determinada por el grado de relación con las diferentes formas y contextos de uso y consumo de la ley.

En efecto, y en el ámbito de la cotidianidad, la ley y el derecho son usados y consumidos a través de diferentes plataformas de emergencia discursiva y narrativa como: Sentencias, documentos académicos, discursos de prensa, libros, conversaciones informales, partes policiales, películas, documentales, conferencias, e infinito, etcétera. Al respecto, Masson y O'connor mencionan:

Justice is a system of representation: Representation of truth by litigating parties in their arguments and embodiment by legal system themselves of their own powers. However the image given is not always the one perceived. the public understanding of law is gleaned from cultural representations of justice which reflects. In fact, popular culture movies, caricatures, portrayal of trials by media or crime fiction shape the image of justice ['La justicia es un sistema de representación: representación de verdades por parte de litigantes en sus argumentos y por el propio sistema legal en sus propios poderes. Sin embargo, la imagen que se da no siempre es la que se percibe. La comprensión pública de la ley se deriva de las representaciones culturales que la justicia refleja. De hecho, las películas de cultura popular, las caricaturas, el retrato de los juicios de los medios o la ficción criminal dan forma a la imagen de la justicia'] (Masson and O'connor, 2007, p. 15).

Esta noción de ley y derecho permite observar como los diversas formas que adquiere el discurso legal traspasan el ámbito de lo estrictamente jurídico, lo que evidencia el impacto social del discurso y las narrativas del derecho y la ley como herramientas de uso y consumo conceptual en la vida cotidiana.

$\mathrm{Al}$ respecto, no se debe olvidar que el discurso jurídico no es un discurso cualquiera, sino que goza de cierta cobertura institucional que lo legitima para la producción y reproducción de verdades y saberes que, posteriormente, darán paso a la construcción de ideologías y representaciones que encuentran fundamentos y justificaciones incuestionables si se invoca la idea de que «la Ley lo dice» o «la Ley define esto como...».

Con todo, se hace clave entender la importancia de los estudios multidisciplinarios del derecho y la ley en la construcción de representaciones sobre lo jurídico; sobre todo teniendo en cuenta que la ley y el derecho bien pueden transformarse - y lo hacen-en instrumentos y prácticas en pro del poder corporativo o particular de ciertos intereses. La Ley, al ser incuestionable y aparentemente objetiva conceptualmente, permite el despliegue de procesos de criminalización, injusticia social u otros mediante su uso y consumo indiscriminado y ausente de reflexión crítica. Así, la ley puede adquirir la forma de un instrumento contrario a su propia esencia de instrumento de justicia. 


\section{Referencias}

Aguilera, R. y González, J. (2011). Derecho, Verdad y Poder en la teoría político-jurídica de Michel Foucault. Revista A Parte Rei. España.

Albornoz, G. (2018). La ley 18.314, y la idea de criminalidad terrorista para distintos actores en su vida cotidiana: expresiones desde la vida cotidiana, de las relaciones entre la construcción ideológica de la ley antiterrorista entendida como discurso, y las representaciones sociales sobre la criminalidad terrorista desde la perspectiva de diversos actores en la Araucania (Tesis de maestría). Temuco, Chile: Universidad de la Frontera.

Althusser, L. (1989). Ideología y aparatos ideológicos del Estado. México: Editorial siglo XXI.

Bascuñan, A. (1997). Tratado de derecho constitucional tomo 1: principios, Estado y Gobierno. Santiago: Editorial Jurídica de Chile.

Bengoa, J. (2001). Historia de un conflicto: El Estado y los Mapuches en el siglo XX. Editorial Planeta: Chile.

Berger, P. y Luckmann, T. (1991). La construcción social de la realidad. Buenos Aires: Amorrortu editores.

Biblioteca del Congreso Nacional (2018). Evolución de la legislación Antiterrorista en Chile y España. Valparaíso: Departamento de estudios, extensión y publicaciones.

De Sousa Santos, B. (2012). Derecho y emancipación. Pensamiento jurídico contemporáneo no 2. Corte Constitucional para el periodo de transición. Quito: Ecuador.

De Certeau, M. (1986). La invención de lo cotidiano. I: Las artes del hacer. México: Universidad Iberoamericana.

Del Valle, C. (2018). Las narrativas del proyecto civilizatorio y la producción del enemigo íntimo en la industria cultural chilena: Modos y estrategia de sujeción. En M. Loayza (Presidencia). Diacronia de la crisis: conflictos, desigualdades y narrativas en los medios de comunicación en Latino América. Conferencia llevada a cabo en Seminario de la Universidad Nacional Mayor de San Marcos. Lima.

Escandón, J. (2008). Curso de introducción al Derecho. Colección de manuales Historia, Filosofía y Ciencia General del Derecho. Concepción: Universidad de Concepción, Chile.

Fish, S. (1995). Is There a Text in this Class? The Authority of Interpretive Communities. Cambridge: Harvard University Press.

Foucault, M. (1996). La verdad y las formas jurídicas. Barcelona: Editorial Gedisa.

Foucault, M. (2003). La Arqueología del saber. Buenos Aires: Editorial Siglo XXI.

Foucault, M. (2014). Defender la sociedad. 60 Edición. Fondo de cultura económica. Buenos Aires.

Gil, J. (1968). Derecho e ideología. Revista de estudios políticos ,157. España.

Giménez, G. (1981). Poder, Estado y Discurso. Perspectivas sociológicas y semiológicas del discurso jurídico y político. Universidad Nacional Autónoma de México. 
Jakobs, G. y Cancio, M. (2003). Derecho Penal del Ciudadano y Derecho Penal del Enemigo. Madrid: Editorial Civitas.

Jodelet, D. (1986). La representación social: fenómenos, concepto y teoría. En Moscovici, Serge (Comp.), Psicología social II 469-494. Barcelona: Editorial Paidós

Jodelet, D. (2008). El movimiento de retorno al sujeto y el enfoque de las representaciones sociales. Revista CONNEXION, 89. Francia: Editorial Érès.

H. Kelsen (1945). General Theory al Law and State. Cambridge, Harvard University Press: Universidad Nacional Autónoma de México.

Masson, A. y O'connor, K. (2007). Representations of Justice. Brussels: P.I.E Peter Lang editions.

Mieles, E. (2004). El concepto de Derecho en Foucault, la Ley y la crítica del paradigma liberal. Revista IUSTA. Conferencia pronunciada en el espacio académico de la Cátedra Fray Bartolomé de las Casas. Colombia.

Nieto, S. (2007). Metodología jurídica e ideologías en el derecho sobre la influencia de la ideología en los ámbitos de aplicación del derecho. Estudios en homenaje a Marcia Muñoz de Alba Medrano. Universidad Autónoma de México. México.

Nandy, A. (1983). El enemigo intimo: Pérdida y recuperación del yo bajo el colonialismo. Oxford University Press: Nueva Delhi.

Nosetto, L. (2015). Max Weber y el concepto de lo político. La ética guerrera y la necesidad de la culpa. Revista Estudios Políticos, 46. Instituto de Estudios Políticos: Universidad de Antioquia.

Riofrío, J. (2013). Lecturas jurídicas de la obra de Santo Tomás de Aquino. Revista Díkaion, 22 pp. 51-81.

Van Dijk, T. (2005a). Ideología y análisis de discurso. Quórum académico (2), 2. Universidad de Zulia: Venezuela.

Van Dijk, T. (2005b). Política, ideología y discurso. Revista Utopia y praxis latinoamericana. Universidad de Zulia: Venezuela.

Van Dijk, T. y Mendizábal, I. (1999). Análisis del discurso social y político. Serie Pluriminor AbyaYala: Quito.

Vilajosana, J. (2010). La construcción social de la realidad jurídica. Revista de ciencias sociales Prisma social, 4. Barcelona. 